\title{
Dual Self-Assembly in Strongly Asymmetric A-B-A Triblock Copolymer Melts Studied by Self-Consistent Field Theory and Monte Carlo Simulations
}

\author{
M. Dzięcielski ${ }^{1}$, S. Wołoszczuk ${ }^{2}$, M. Banaszak $^{3 *}$ \\ ${ }^{1}$ Faculty of Geographical and Geological Sciences, Adam Mickiewicz University \\ ul. Krygowskiego 10, 61-680 Poznań, Poland \\ ${ }^{2}$ Faculty of Physics, Adam Mickiewicz University \\ ul. Umultowska 85, 61-614 Poznan, Poland \\ ${ }^{3 *}$ Faculty of Physics and NanoBioMedical Centre, Adam Mickiewicz University \\ ul. Umultowska 85, 61-614 Poznan, Poland \\ ${ }^{*}$ E-mail:mbanasz@amu.edu.pl
}

Received: 28 November 2018; revised: 12 December 2018; accepted: 14 December 2018; published online: 24 December 2018

\begin{abstract}
Using the Self-Consistent Field Theory (SCFT) we study the dual self-assembly of ABA triblock copolymers melts and compare the numerical results with those obtained by the lattice Monte Carlo simulations. While the results are qualitatively similar for both methods, the simulation times are significantly shorter for the SCFT calculations than those for the corresponding Monte Carlo simulations.
\end{abstract}

Key words: triblock copolymer, self-consistent field theory, Monte Carlo simulation, self-assembly

\section{INTRODUCTION}

Self-assembly in the block copolymer melts is an active area of research [1-16]. Block copolymers self-assemble via the microphase separation which is an instant of the orderdisorder transition (ODT). This transition leads to formation of a plethora of different nanostructures, such as ordered layers, hexagonally arranged cylinder, cubically ordered spheres or a double gyroid phase. Previous studies [17-23] of molecularly asymmetric A1BA2 triblock copolymers synthesized from a parent diblock copolymer so that $N_{A 1}$ is significantly smaller than $N_{A 2}$ (where $N_{A 1}$ and $N_{A 2}$ denote the number of repeat units in the A1 and A2 blocks, respectively) have helped to elucidate the molecular and property changes accompanying the transformation from an $\mathrm{AB}$ diblock to a molecularly symmetric ABA triblock copolymer (with $N_{A 1}=N_{A 2}$ ). Recent Monte Carlo (MC) simulations of moderately segregated copolymers have yielded results that quantitatively agree with unexpected experimental findings, most notably a pronounced minimum in the order-disorder transition temperature as $N_{A 2}$ is progressively increased.

In the limit of superstrong segregation (SSS) [24, 25], interstitial micelles composed of the minority A2 endblock are observed to be arranged into two-dimensional hexagonal arrays along the midplane of B-rich lamellae in compositionally symmetric (50:50 A:B) copolymers. Calculations performed here establish the coupled molecular-asymmetry and incompatibility conditions under which such micelles form. Beyond an optimal length of the A2 endblock, the propensity for interstitial micelles to develop decreases, and the likelihood for colocation of both endblocks in the A 1 -rich lamellae increases. The SSS regime has also been studied experimentally [26-30] and nontraditional morphologies have been observed [27, 28, 31]. 
From previous studies [17-23] we know that as the A1BA2 triblock melt begins to self-organize, short A-blocks initially locate in the B-domain, and then as the $\chi$ value grows they migrate to A-domain. Moreover, for sufficiently long chains and relatively high $\chi$ values we can observe that short A-segments inside the B-domain aggregate into interstitial micelles (IM's).

This observation will be investigated by the selfconsistent field theory (SCFT) and reaffirmed by lattice Monte Carlo methods, which are described in the following section.

\section{METHODS}

\section{1. SCFT Method}

The incompressible ABA copolymer melt is modeled as a collection of $n$ diblock chains confined in volume $V$. Each chain, labeled $\alpha=1,2, \ldots, n$, can take any Gaussian configuration parameterized from $s=0$ to $s=f_{1}$ for $A$-segments, from $s=f_{1}$ to $s=f_{2}$ for $B$-segments, and from $s=f_{2}$ to $s=1$ for $A$-segments. Up to a multiplicative constant, the partition function for a single Gaussian chain in external fields $W_{A}(\mathbf{r})$ and $W_{B}(\mathbf{r})$ acting on segments $\mathrm{A}$ and $\mathrm{B}$, respectively, is

$$
\begin{gathered}
\mathcal{Q}\left[W_{A}, W_{B}\right] \equiv \int \tilde{\mathcal{D}} \mathbf{r}_{\alpha}(\cdot) \exp \left[-\int_{0}^{f} d s W_{A}\left(\mathbf{r}_{\alpha}(s)\right)\right. \\
\left.-\int_{f_{1}}^{f_{2}} d s W_{B}\left(\mathbf{r}_{\alpha}(s)\right)-\int_{f_{2}}^{1} d s W_{A}\left(\mathbf{r}_{\alpha}(s)\right)\right]
\end{gathered}
$$

The path integral, $\int \tilde{\mathcal{D}} \mathbf{r}_{\alpha}(\cdot)$, is taken over single-chain trajectories, $\mathbf{r}_{\alpha}(s)$, with Wiener measure expressed as $\tilde{\mathcal{D}} \mathbf{r}_{\alpha}=$ $\mathcal{D} \mathbf{r}_{\alpha} P\left[\mathbf{r}_{\alpha} ; 0,1\right]$, and

$$
P\left[\mathbf{r}_{\alpha} ; s_{1}, s_{2}\right] \propto \exp \left[-\frac{3}{2 N a^{2}} \int_{s_{1}}^{s_{2}} d s\left|\frac{d}{d s} \mathbf{r}_{\alpha}(s)\right|^{2}\right]
$$

Note that $a$ is the segment size, and $N a^{2}$ is the mean squared end-to-end distance of a Gaussian chain. By Kac-Feynman theorem, eq 1 can be related to a Fokker-Planck partial differential equation, known also as a modified diffusion equation (MDE) and shown with appropriate details below (eqs 15 and 16).

Segments $A$ and $B$ interact via the $\chi$ parameter which provides an effective measure of incompatibility between them. Evaluation of the full partition function of $n$ interacting diblock chains, shown below (eq 3 ), is a highly challenging task, involving many-body interactions, both intermolecular and intramolecular.

$$
Z=\int \prod_{\alpha=1}^{n} \tilde{\mathcal{D}} \mathbf{r}_{\alpha} \delta\left[1-\hat{\phi}_{A}-\hat{\phi}_{B}\right] \exp \left[-\chi \rho_{0} \hat{\phi}_{A} \hat{\phi}_{B}\right],
$$

where $\delta$-function enforces incompressibility (the melt is assumed to be incompressible), and

$$
\begin{aligned}
\hat{\phi}_{A}(\mathbf{r}) & =\frac{N}{\rho_{0}} \sum_{\alpha=1}^{n}\left[\int_{0}^{f_{1}} d s \delta\left(\mathbf{r}-\mathbf{r}_{\alpha}(s)\right)\right. \\
& \left.+\int_{f_{2}}^{1} d s \delta\left(\mathbf{r}-\mathbf{r}_{\alpha}(s)\right)\right]
\end{aligned}
$$

$$
\hat{\phi}_{B}(\mathbf{r})=\frac{N}{\rho_{0}} \sum_{\alpha=1}^{n} \int_{f_{1}}^{f_{2}} d s \delta\left(\mathbf{r}-\mathbf{r}_{\alpha}(s)\right)
$$

are the microscopic segments densities of $A$ and $B$, respectively; $\rho_{0}=n N / V$ is the segment number density. After replacing microscopic segment (or particle) densities with a variety of fields, by inserting and spectrally decomposing the appropriate $\delta$-functionals, the partition function of an incompressible diblock melt is

$$
\begin{aligned}
Z= & \mathcal{N} \int \mathcal{D} \phi_{A}(\cdot) \mathcal{D} W_{A}(\cdot) \mathcal{D} \phi_{B}(\cdot) \mathcal{D} W_{B}(\cdot) \mathcal{D} \Psi(\cdot) \\
& \exp \left[-\frac{F\left[\phi_{A}, W_{A}, \phi_{B}, W_{B}, \Psi\right]}{k_{B} T}\right],
\end{aligned}
$$

where $\mathcal{N}$ is a normalization factor. The functional integral is taken over the relevant fields $\phi_{A}(\mathbf{r}), W_{A}(\mathbf{r}), \phi_{B}(\mathbf{r}), W_{B}(\mathbf{r})$, and $\Psi(\mathbf{r})$, with the free energy functional, $F\left[\phi_{A}, W_{A}, \phi_{B}, W_{B}, \Psi\right]$, including the single chain partition function (in external fields $W_{A}(\mathbf{r})$ and $W_{B}(\mathbf{r})$ ), as shown below

$$
\begin{aligned}
& \frac{F\left[\phi_{A}, W_{A}, \phi_{B}, W_{B}, \Psi\right]}{n k_{B} T} \equiv \\
\equiv & -\ln \frac{\mathcal{Q}}{V}+V^{-1} \int d \mathbf{r}\left[N \chi \phi_{A}(\mathbf{r}) \phi_{B}(\mathbf{r})\right. \\
- & W_{A}(\mathbf{r}) \phi_{A}(\mathbf{r})-W_{B}(\mathbf{r}) \phi_{B}(\mathbf{r}) \\
- & \left.\Psi(\mathbf{r})\left(1-\phi_{A}(\mathbf{r})-\phi_{B}(\mathbf{r})\right)\right]
\end{aligned}
$$

Fields $\phi_{A}(\mathbf{r})$ and $\phi_{B}(\mathbf{r})$ are associated with normalized concentration profiles of $A$ and $B$, and fields $W_{A}(\mathbf{r})$ and $W_{B}(\mathbf{r})$ with chemical potential fields acting on $A$ and $B$, respectively; field $\Psi(\mathbf{r})$ enforces incompressibility. Evaluating functional integrals in eq 6 is a challenging task which, in principle, can be performed by field theoretic simulations. A simpler, but approximate, approach is based on the mean-field idea, where the dominant and, in fact, only contribution to the functional integral in eq 6 comes from the fields satisfying the saddle point condition expressed as the following set of equations:

$$
\frac{\delta F}{\delta \phi_{A}}=\frac{\delta F}{\delta \phi_{B}}=\frac{\delta F}{\delta W_{A}}=\frac{\delta F}{\delta W_{B}}=\frac{\delta F}{\delta \Psi}=0
$$


Performing the above functional derivatives yields

$$
\begin{aligned}
W_{A}(\mathbf{r}) & =N \chi \phi_{B}(\mathbf{r})+\Psi(\mathbf{r}) \\
W_{B}(\mathbf{r}) & =N \chi \phi_{A}(\mathbf{r})+\Psi(\mathbf{r}) \\
1 & =\phi_{A}(\mathbf{r})+\phi_{B}(\mathbf{r}) \\
\phi_{A}(\mathbf{r}) & =\frac{V}{\mathcal{Q}}\left[\int_{0}^{f_{1}} d s q(\mathbf{r}, s) q^{\dagger}(\mathbf{r}, s)\right. \\
& \left.+\int_{f_{2}}^{1} d s q(\mathbf{r}, s) q^{\dagger}(\mathbf{r}, s)\right] \\
\phi_{B}(\mathbf{r}) & =\frac{V}{\mathcal{Q}} \int_{f_{1}}^{f_{2}} d s q(\mathbf{r}, s) q^{\dagger}(\mathbf{r}, s),
\end{aligned}
$$

where $\mathcal{Q} / V$ can be calculated as

$$
\frac{\mathcal{Q}}{V}=\frac{1}{V} \int d \mathbf{r} q(\mathbf{r}, 1)
$$

and $q(\mathbf{r}, s)$ is the forward chain propagator which is the solution of the following modified diffusion equation

$$
\begin{aligned}
& \frac{\partial q}{\partial s}=\frac{1}{6} N a^{2} \nabla^{2} q-W_{A}(\mathbf{r}) q, \quad 0 \leq s \leq f_{1} \\
& \frac{\partial q}{\partial s}=\frac{1}{6} N a^{2} \nabla^{2} q-W_{B}(\mathbf{r}) q, \quad f_{1} \leq s \leq f_{2} \\
& \frac{\partial q}{\partial s}=\frac{1}{6} N a^{2} \nabla^{2} q-W_{A}(\mathbf{r}) q, \quad f_{2} \leq s \leq 1
\end{aligned}
$$

with the initial condition $q(\mathbf{r}, 0)=1$. Similarly $q^{\dagger}(\mathbf{r}, s)$ is the backward chain propagator which is the solution of the conjugate modified diffusion equation:

$$
\begin{aligned}
-\frac{\partial q^{\dagger}}{\partial s} & =\frac{1}{6} N a^{2} \nabla^{2} q^{\dagger}-W_{A}(\mathbf{r}) q^{\dagger}, \quad 0 \leq s \leq f_{1} \\
-\frac{\partial q^{\dagger}}{\partial s} & =\frac{1}{6} N a^{2} \nabla^{2} q^{\dagger}-W_{B}(\mathbf{r}) q^{\dagger}, \quad f_{1} \leq s \leq f_{2} \\
-\frac{\partial q^{\dagger}}{\partial s} & =\frac{1}{6} N a^{2} \nabla^{2} q^{\dagger}-W_{A}(\mathbf{r}) q^{\dagger}, \quad f_{2} \leq s \leq 1
\end{aligned}
$$

with the initial condition $q^{\dagger}(\mathbf{r}, 1)=1$.

While the set of equations $9,10,11,12$, and 13 can be solved, in principle, in a self-consistent manner, it is difficult to solve this set without some additional assumptions. First, we assume that the melt forms a spatially ordered nanophase. Second, we use the UCA which is a considerable simplification, limiting our attention to a single D-dimensional spherical cell of radius $R$, and volume $V$. All fields within this cell have radial symmetry, which reduces this problem computationally to a single radial coordinate, $r$. Thus eq 14 can be rewritten as

$$
\frac{\mathcal{Q}}{V}=D \frac{\int_{0}^{R} r^{D-1} q(r, 1) d r}{R^{D}}
$$

Note that the factor, $D$, in front of the above integral originates from the ratio of the area of a sphere with radius 1 to the volume of a spherical cell with the same radius, both in $D$ dimensions.

While in integrals (eqs 12,13 and 14) we replace $\mathbf{r}$ with $r$, and $d \mathbf{r} / V$ with $D r^{D-1} d r / R^{D}$, in the modified diffusion equations, 15 and 16, we replace $\mathbf{r}$ with $r$ and use the spherically symmetric form of the Laplacian

$$
\nabla^{2} f=\frac{\partial^{2} f}{\partial r^{2}}+\frac{D-1}{r} \frac{\partial f}{\partial r}
$$

and similarly, in equations for both propagators $q(r, s)$ and $q^{\dagger}(r, s)$, we replace $\mathbf{r}$ with $r$. Obviously the solution depends on radius, $R$, and dimensionality, $D=1,2$ and 3, corresponding to 3 different nanophases. We use the CrankNicholson scheme to solve iteratively the modified diffusion equations (eqs 15 and 16) in their radial form, until the selfconsistency condition is met, obtaining the saddle point fields, $\overline{\phi_{A}}(r), \overline{\phi_{B}}(r), \overline{W_{A}}(r)$ and $\overline{W_{B}}(r)$ for a given $R$ and $D$. In the MF approximation, the free energy functional becomes the free energy, and therefore we calculate the reduced free energy (per chain in $k_{B} T$ units) by substituting the saddle point fields into eq 7 :

$$
\begin{aligned}
\frac{F(R, D)}{n k_{B} T} \equiv & -\ln \frac{\mathcal{Q}}{V}+\frac{D}{R^{D}} \int_{0}^{R} r^{D-1}\left[N \chi \overline{\phi_{A}}(r) \overline{\phi_{B}}(r)-\right. \\
& \left.\overline{W_{A}}(r) \overline{\phi_{A}}(r)-\overline{W_{B}}(r) \overline{\phi_{B}}(r)\right] d r
\end{aligned}
$$

Since in the MF theory, the stability of a nanophase depends on the product $\chi N$ and composition, $f$, we start, at a given point of the phase diagram, $(f, \chi N)$, with numerical calculation of $F(R, D)$ (eq 19) for various $D$ 's $(1,2$, and 3 ) and $R$ 's. In order to solve the MDE's (eqs 15 and 16) we use up to $N_{T}=1000$ and up to $N_{R}=2000$ steps for the "time", $s$, and space, $r$, variables, respectively.

Numerically, we find $R$ and $D$ which minimize $F(R, D)$, and this allows us to determine the dimensionality, $D$, of the most stable nanophase, and therefore the most favorable nanophase itself. But the free energy of this nanophase has to be compared to that of the disordered phase. Therefore, we calculate the difference

$$
\frac{\Delta F}{n k_{B} T} \equiv \frac{F}{n k_{B} T}-\frac{F_{d i s}}{n k_{B} T}
$$

where $F_{d i s}$ is the free energy of the disordered phase:

$$
\frac{F_{d i s}}{n k_{B} T}=N \chi f(1-f)
$$

If $\Delta F$ is negative then the appropriate nanophase is thermodynamically stable for the point considered, $(f, \chi N)$; otherwise the system is the disordered phase. 


\section{2. MC Method}

The cooperative motion algorithm (CMA) [32-36], based on FCC lattice, is used to simulate the triblock solutions. We apply standard Monte Carlo (MC) simulations with the Metropolis algorithm [37] as well as parallel tempering (PT) method [38-40]. In the PT case, $M$ replicas of system are simulated in parallel, each in different temperature $T_{i}$, with $i$ ranging from 1 to $M$. After $3000 \mathrm{MC}$ steps we try to exchange replicas with neighboring $T_{i}$ in random order with probability:

$$
\begin{aligned}
& p\left(T_{i} \leftrightarrow T_{i+1}\right)= \\
= & \min \left[1, \exp \left(-\left(\beta_{i}-\beta_{i+1}\right)\left(U_{i+1}-U_{i}\right)\right)\right],
\end{aligned}
$$

where $\beta_{i}=1 / k T_{i}$ and $U_{i}$ is potential energy of replica at $T_{i}$. This method offers efficient equilibration at low temperatures.

We repeat the experiment at least 3 times starting with different initial configurations, in which the polymer chains assume statistical conformations and random orientations, and are uniformly distributed within the simulation box. A single MC step is defined as an attempt to move a given segment. Usually, the first half of run is used to equilibrate the system and the second one to collect the data. The results are averaged over all simulation runs. While the morphologies obtained in the simulations were quantitatively dependent on the box size, this dependence was weak and did not change the main conclusions qualitatively.

We use the following set of interaction energies which are limited to the nearest neighbors:

$$
\begin{aligned}
& \epsilon_{A B}=\epsilon \\
& \epsilon_{A A}=0 \\
& \epsilon_{B B}=0,
\end{aligned}
$$

where $\epsilon$ is an energy unit, and we define the reduced energy per lattice site and the reduced temperature as:

$$
\begin{gathered}
\frac{E^{*}}{n_{a}}=\frac{E / \epsilon}{n_{a}} \\
T^{*}=\frac{k T}{\epsilon}
\end{gathered}
$$

On the basis of considerations presented in reference [41] we can relate $T^{*}$ used in this paper to the Flory $\chi$ parameter employed in the self-consistent field theory by the following approximate equation:

$$
\chi=\frac{7.5}{T^{*}}
$$

The above equation can also be used in order to relate theoretical $T^{*}$ 's to experimental $\chi$ 's.

\section{RESULTS}

Monte Carlo simulations were carried out using the cooperative motion algorithm (CMA) for the following chain lengths: $\mathrm{N}=32,64,80,96$ and 112. In each case, the ratio of segments $A$ and $B$ is $1: 1$. For each chain length, the number of monomers in the short A1-block is $N_{A 1}=1,2,3$ and 4 , respectively. The SCFT calculations were performed for the corresponding parameters.

For the $N=32$ chain we used the $32 \times 32 \times 32$ and $64 \times 32 \times 32$ simulation boxes, for the $N=64$ chain the $64 \times 32 \times 32$ and $64 \times 64 \times 64$ boxes, for the $N=80$ chain the $80 \times 40 \times 40$ box, for the $N=96$ chain the $96 \times 48 \times 48$ box, and for the $N=112$ chain the $112 \times 56 \times 56$ box.

We determined the order-disorder (ODT) $\chi$ 's (and thus temperatures). We also introduce interface-domain transition (IDT) $\chi$ 's as those in which half of the dangles are within B-domain and half in A-domain (interface). The $\chi N_{I D T}$ 's describes quantitatively the process of migration of short A1's from B-domain to the native A-domain. $\chi N_{I D T}$ in SCFT calculations are determined from short A1's density profiles in the direction normal to the layers, whereas in Monte Carlo simulations they are determined directly from the configurations. In addition we determine the $\chi$ range in which aggregation into interstitial micelles (IM) within B-domain occurs.

In Fig. 1 triangles refer to SCFT calculations, while squares and diamonds to MC results. Open symbols denote ODT, solid triangles and squares refer to IDT, while solid diamonds and dashed lines indicate the area, where the IM's are observed.

In Fig. 1(a) we can see $\chi N_{O D T}$ in the function of $f_{A 1}$ (open symbols) and $\chi N_{I D T}$ (closed symbols). Above $\chi N_{O D T}$, most of the short A1's are in the B-domain. As $\chi$ grows A1's share in the B-domain and A-domain gets even (IDT line). Then, for the larger $\chi$ values, the share of A1's in domain $\mathrm{A}$ is dominant.

In Fig. 1(b) we add the Monte Carlo simulation results, where open squares mean $\chi N_{O D T}$ while solid squares refer to $\chi N_{I D T}$. We can see clearly that both qualitatively and quantitatively SCFT calculations and Monte Carlo simulations give consistent results when it comes to determining order-disorder transition and interface-domain transitions.

In Fig. 1(c), we additionally apply the information about the area in which we observe the aggregation of short A1's into micelles within the B-domain (solid diamonds and dashed lines).

We can see clearly that as $f_{A 1}$ grows (e.g. shorter chains) the temperature range of the occurrence of IM's becomes narrow but moves towards higher (more easily accessible) temperatures (lower $\chi$ 's). On the other hand, when the value of $f_{A 1}$ decreases and we deal with this by maintaining the length of A1-block while increasing the length of the chain, $N$, the $\chi$ range in which the aggregation occurs is wider, but shifted towards higher $\chi$ values, i.e. lower temperatures. 

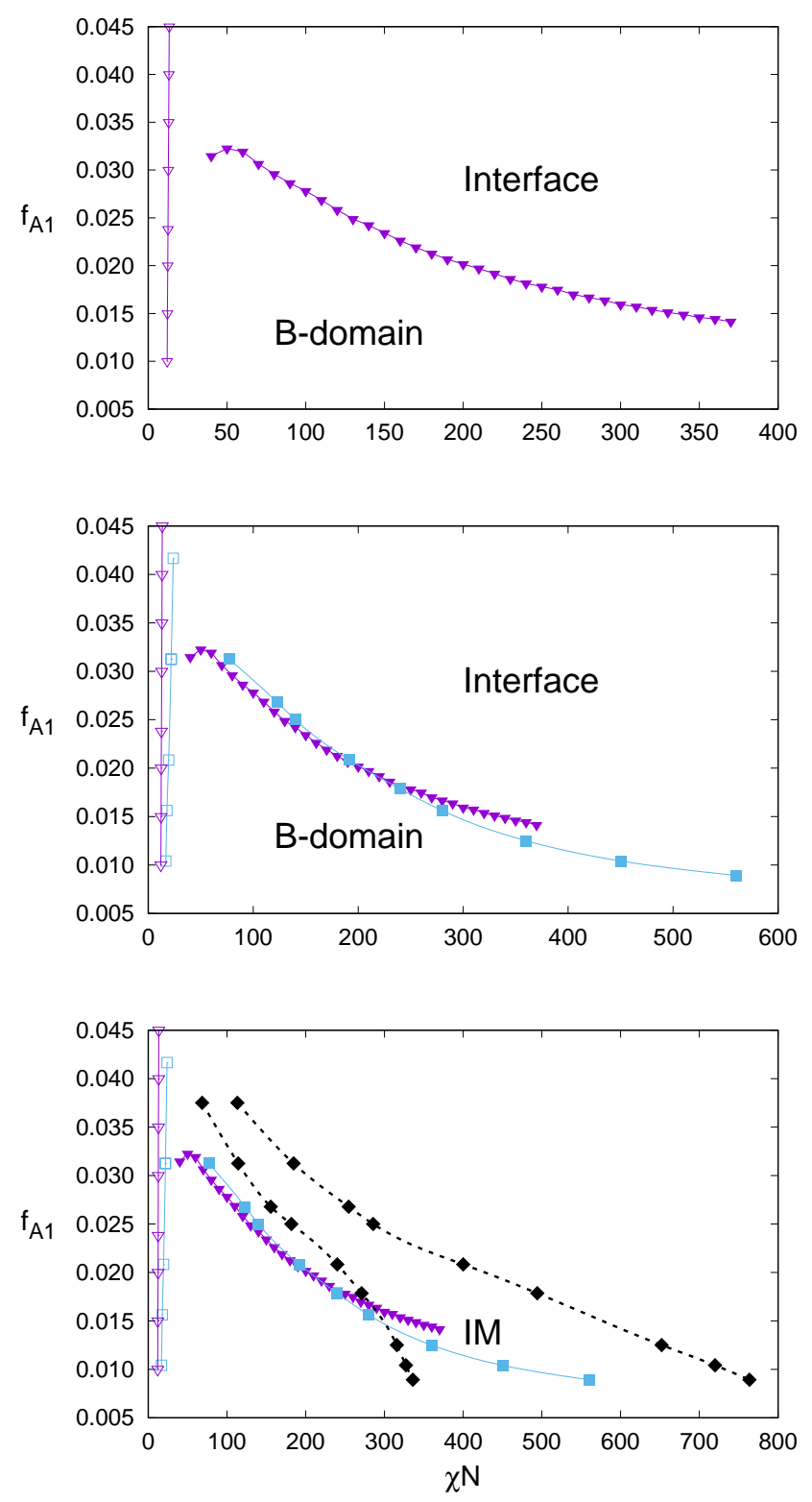

Fig. 1. Diagram containing information on the order-disorder transition (ODT), location of shorter A1-blocks within individual domains, and aggregation of A1-blocks into micelles for molten A1-B-A2 triblock copolymer in the function Flory parameter, $\chi$ : (a) ODT (open triangles) and interface-domain transition, IDT (solid triangles), from SCFT calculations; (b) SCFT results with Monte Carlo ODT (open squares) and IDT (solid squares) lines; (c) the area in which interstitial micelles (IM) has been observed (solid diamonds and dashed lines) against the background of results shown in (a) and (b).

In Fig. 2 we present an MC snapshot of molten 2(A1)48(B)-46(A2) copolymer. The A-domain is marked in blue and the short A1's in yellow. B-domain is not shown for clarity purposes. Red color denotes B-segments of these chains whose A1's form one of the micelles within the B-domain. We use a recursive algorithm which allows us to determine whether a given chain forms a bridge, a loop, or is part of an IM.

Finally, it is also worthwhile underlining that the differences in results obtained by these two methods are relatively small. This can be attributed to the fact that the mean-field approach, such as the SCFT, is known to work very well in the dense polymer systems. These small differences are usually explained in terms a large coordination number, which scales $\sqrt{N}$, where $N$ is large for polymers, suppressing fluctuations as argued by Fredrickson [42]. 


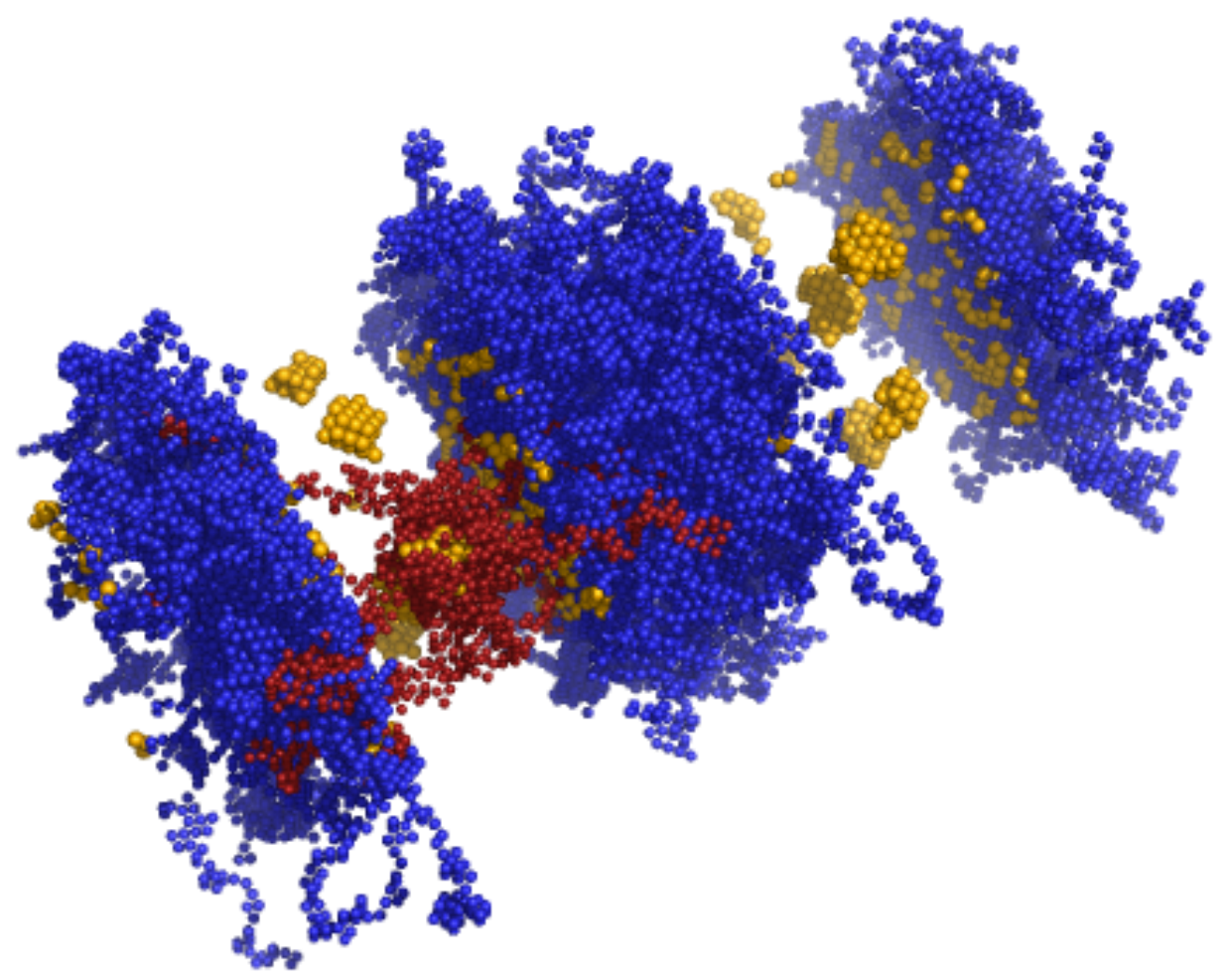

Fig. 2. Monte Carlo snapshot of 2(A1)-48(B)-46(A2) triblock copolymer melt. Domain A is marked in blue. Short A-segments (A1) marked in yellow. B-domain is not shown for clarity. The red segments are the B-segments of these chains, which form a single micelle inside the $\mathrm{B}$ domain.

\section{CONCLUSIONS}

Using the Self-Consistent Field Theory (SCFT) we study the dual self-assembly of ABA triblock copolymers melts and compare the numerical results with those obtained by the lattice Monte Carlo simulations. While the results are qualitatively similar for both methods, the simulation times are significantly shorter for the SCFT calculations than those for the corresponding Monte Carlo simulations. It is also worthwhile underlining that the differences in results obtained by these two methods are relatively small. This can be attributed to the fact that the mean-field approach, such as the SCFT, is known to work very well in the dense polymer systems. These small differences are usually explained in terms of a large coordination number, which scales as $\sqrt{N}$, where $N$ is large for polymers, suppressing fluctuations.

\section{Acknowledgments}

We gratefully acknowledge the computational grant from the Supercomputing and Networking Center (PCSS) in Poznan and the Polish National Science Centre (Grant NCN No. UMO-2017/25/B/ST5/01970).

\section{References}

[1] I.W. Hamley The Physics of Block Copolymers, 1998.

[2] M. Lazzari, G. Liu, S. Lecommandoux, Block Copolymers in Nanoscience, pp. 1-428. Block Copolymers in Nanoscience, 2008.

[3] V. Abetz, P.F.W. Simon, Phase behaviour and morphologies of block copolymers, Advances in Polymer Science, 1892005.

[4] A.N. Semenov Sov.Phys.JETP 61, 733-742 (1985).

[5] F.S. Bates G.H. Fredrickson, Block copolymers-designer soft materials, Physics Today 52(2), 32-38 (1999).

[6] C. Park, J. Yoon, E.L. Thomas, Enabling nanotechnology with self assembled block copolymer patterns, Polymer 44(22), 6725-6760 (2003).

[7] V. Abetz, Block copolymers, ternary triblocks, Encyclopedia of Polymer Science and Technology 1, 482-523 (2003).

[8] Z. Guo, G. Zhang, F. Qiu, H. Zhang, Y. Yang, A. . Shi, Discovering ordered phases of block copolymers: New results from a generic fourier-space approach, Physical Review Letters 101(2) (2008).

[9] M.W. Matsen, R.B. Thompson, Equilibrium behavior of symmetric aba triblock copolymer melts, Journal of Chemical Physics 111(15), 7139-7146 (1999).

[10] M.W. Matsen, Equilibrium behavior of asymmetric aba triblock copolymer melts, Journal of Chemical Physics 113(13), 5539-5544 (2000).

[11] P. Alexandridis, U. Olsson, B. Lindman, A record nine different phases (four cubic, two hexagonal, and one lamellar lyotropic liquid crystalline and two micellar solutions) in a 
ternary isothermal system of an amphiphilic block copolymer and selective solvents (water and oil), Langmuir 14(10), 2627-2638 (1998).

[12] A.S. Krishnan, S.D. Smith, R.J. Spontak, Ternary phase behavior of a triblock copolymer in the presence of an endblockselective homopolymer and a midblock-selective oil, Macromolecules 45(15), 6056-6067 (2012).

[13] M.W. Matsen, F.S. Bates, Origins of complex self-assembly in block copolymers, Macromolecules 29(23), 7641-7644 (1996).

[14] J.T. Chen, E.L. Thomas, C.K. Ober, G. . Mao, Self-assembled smectic phases in rod-coil block copolymers, Science 273, 343-346 (1996).

[15] J. Raez, I. Manners, M.A. Winnik, Nanotubes from the selfassembly of asymmetric crystalline-coil poly(ferrocenylsilanesiloxane) block copolymers, Journal of the American Chemical Society, 124(35), 10381-10395 (2002).

[16] M.W. Matsen, M. Schick, Lamellar phase of a symmetric triblock copolymer, Macromolecules 27(1), 187-192 (1994).

[17] M.W. Hamersky, S.D. Smith, A.O. Gozen, R.J. Spontak, Phase behavior of triblock copolymers varying in molecular asymmetry, Physical Review Letters 95(16), (2005).

[18] S. Woloszczuk, M. Banaszak, R.J. Spontak, Monte-carlo simulations of the order-disorder transition depression in aba triblock copolymers with a short terminal block, Journal of Polymer Science, Part B: Polymer Physics 51(5), 343-348 (2013).

[19] S.S. Tallury, K.P. Mineart, S. Woloszczuk, D.N. Williams, R.B. Thompson, M.A. Pasquinelli, M. Banaszak, R.J. Spontak, Communication: Molecular-level insights into asymmetric triblock copolymers: Network and phase development, Journal of Chemical Physics 141(12), (2014).

[20] S. Woloszczuk, K.P. Mineart, R.J. Spontak, M. Banaszak, Dual modes of self-assembly in superstrongly segregated bicomponent triblock copolymer melts, Physical Review E Statistical, Nonlinear, and Soft Matter Physics 91(1), (2015).

[21] S. Woloszczuk, S. Jurga, M. Banaszak, Towards entropydriven interstitial micelles at elevated temperatures from selective a1-b-a2 triblock solutions, Physical Review E 94(2), (2016).

[22] S. Woloszczuk, M.O. Tuhin, S.R. Gade, M.A. Pasquinelli, M. Banaszak, R.J. Spontak, Complex phase behavior and network characteristics of midblock-solvated triblock copolymers as physically cross-linked soft materials, ACS Applied Materials and Interfaces 9(46), 39940-39944 (2017).

[23] S. Woloszczuk, M. Banaszak, Interstitial micelles in binary blends of aba triblock copolymers and homopolymers, Physical Review E 97(1), (2018).

[24] I.A. Nyrkova, A.R. Khokhlov, M. Doi, Microdomains in block copolymers and multiplets in ionomers: Parallels in behavior, Macromolecules 26(14), 3601-3610 (1993).

[25] A.N. Semenov, I.A. Nyrkova, A.R. Khokhlov, Polymers with strongly interacting groups: Theory for nonspherical multiplets, Macromolecules 28(22), 7491-7500 (1995).
[26] C. Burger, M.A. Micha, S. Oestreich, S. Förster, M. Antonietti, Characterization of two new stable block copolymer mesophases by synchrotron small-angle scattering, Europhysics Letters 42(4), 425-429 (1998).

[27] T.P. Lodge, M.A. Hillmyer, Z. Zhou, Y. Talmon, Access to the superstrong segregation regime with nonionic abc copolymers, Macromolecules 37(18), 6680-6682 (2004).

[28] R.R. Taribagil, M.A. Hillmyer, T.P. Lodge, Hydrogels from aba and abc triblock polymers, Macromolecules 43(12), 53965404 (2010).

[29] S. Qin, H. Li, W. Yuan, Y. Zhang, Hierarchical self-assembly of fluorine-containing diblock copolymer:from onion-like nanospheres to superstructured microspheres, Polymer 52(4), 1191-1196 (2011).

[30] J. . Deng, W. . Wang, Z. . Zheng, X. . Ding, Y. . Peng, Selfassembly behavior of copolymers with super segregated structure containing fluorinated segments, Chinese Journal of Polymer Science (English Edition) 32, 817-822 (2014).

[31] D.J. Pochan, Z. Chen, H. Cui, K. Hales, K. Qi, K.L. Wooley, Toroidal triblock copolymer assemblies, Science 306(5693), 94-97 (2004).

[32] A. Gauger, A. Weyersberg, T. Pakula, Monte carlo studies of static properties of interacting lattice polymers with the cooperative-motion algorithm, Die Makromolekulare Chemie, Theory uSimulations 2(4), 531-560 (1993).

[33] A. Weyersberg, T.A. Vilgis, Phase transitions in diblock copolymers: Theory and monte carlo simulations, Physical Review E 48(1), 377-390 (1993).

[34] T. Pakula, K. Karatasos, S.H. Anastasiadis, G. Fytas, Computer simulation of static and dynamic behavior of diblock copolymer melts, Macromolecules 30(26), 8463-8472 (1997).

[35] T. Pakula, Simulations on the completely occupied lattice, in Simulation Methods for Polymers (M.J. Kotelyanskii, D.N. Thedorou, eds.), ch. 5, Marcel-Dekker, 2004.

[36] M. Banaszak, S. Woloszczuk, T. Pakula, S. Jurga Phys. Rev. E 66(031804), (2003).

[37] N. Metropolis, A.W. Rosenbluth, M.N. Rosenbluth, A.H. Teller J. Chem. Phys. 21(1087), (1953).

[38] R.H. Swendsen, J.S. Wang, Replica monte carlo simulation of spin-glasses, Physical Review Letters 57(12), 2607-2609 (1986).

[39] D.J. Earl, M.W. Deem, Parallel tempering: Theory, applications, and new perspectives, Physical Chemistry Chemical Physics 7(23), 3910-3916 (2005).

[40] A. Sikorski, Properties of star-branched polymer chains. application of the replica exchange monte carlo method, Macromolecules 35(18), 7132-7137 (2002).

[41] P. Knychała, M. Dzięcielski, M. Banaszak, N.P. Balsara, Phase behavior of ionic block copolymers studied by a minimal lattice model with short-range interactions, Macromolecules 46(14), 5724 (2013).

[42] G. Fredrickson, The Equilibrium Theory of Inhomogeneous Polymers. 2007. 

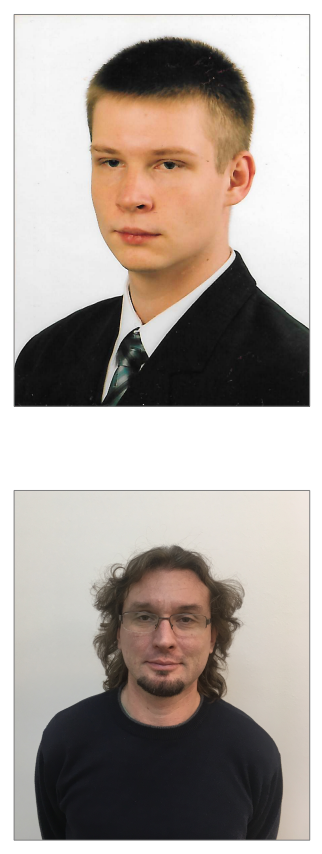

Sebastian Wołoszczuk graduated in 2003 from Adam Mickiewicz University in Poznań, received his PhD degree in Physics in 2008 from the Adam Mickiewicz University in Poznań. Since 2009 he has been working at the Faculty of Physics at the Adam Mickiewicz University. In 2018, he completed a six-month research visit at the Joint Institute of Nuclear Research in Dubna, Russia, where he was involved in molecular dynamics simulations of carbon nanotube-drug systems. In the same year, he became the head of computer laboratories at the Faculty of Physics. His main scientific interests are focused on the physics of soft matter, in particular polymer systems, colloids, self-organization processes, complex systems, computer simulations using Monte Carlo and Molecular Dynamics methods, and developing tools for computer modeling. His interests also include building and administering large-scale computers.

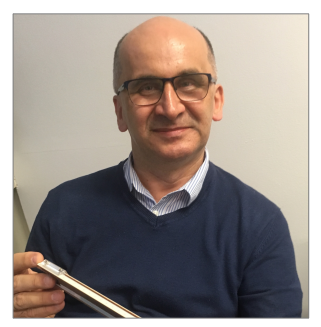

Michał Banaszak graduated in Theoretical Physics in 1985 from the Adam Mickiewicz University in Poznań. He received his PhD degree in Physics in 1991 from Memorial University in St. John's, Canada, specializing in Polymer Physics. From 1992 to 1995 he worked as postdoctoral fellow in Exxon Research \& Engineering Co. in Annandale, New Jersey, USA, working on theory and modeling of polymer solutions. From 1995 to 1997 he worked in the Chemistry Department of UMIST in Manchester, UK, as a research associate, specializing in large-scale computer simulations of ionic copolymers. In 1997, he joined the Adam Mickiewicz University, obtaining his DSc degree (habilitation) in 2004 in Physics (specialty: Soft Matter Physics and Computer Simulations), and later professorship in Physics. His main interest is in developing new models and theories for nanoscale self-assembly of various polymer systems. He also collaborates with experimentalists, using large-scale computing. 